\title{
Surface Material Segmentation Using Polarisation
}

\author{
Nitya Subramaniam and Edwin Hancock \\ Department of Computer Science, University of York, YO10 5DD, United Kingdom \\ \{nitya, erh\}@cs.york.ac.uk
}

\begin{abstract}
This paper describes the use of polarisation information for surface segmentation based on material characteristics. We work with both polarised and unpolarised light, and hence domains where the polarisation is either specular or diffuse. We commence by using moments to estimate the components of the polarisation image (mean-intensity, polarisation degree and phase) from images obtained through multiple polariser orientations. From the Fresnel theory, the phase of light remitted from a surface is equal to the azimuth angle of the remitted direction, and for materials with restricted ranges of refractive index the polarisation degree determines the zenith angle. Based on this observation, we parameterise the angular distribution of the mean intensity for remitted light using spherical harmonics. We explore how vectors of spherical harmonics can be used to characterise varying surface reflectance distributions, and segment a scene into different material patches using Mahalanobis distances and normalized graph cuts.
\end{abstract}

\section{Introduction}

The polarisation of light is used by some animals (e.g. mantis shrimp) to augment the information contained in the visible spectrum. Although humans are insensitive to polarisation, it is a useful addition to colour and intensity information for computer vision applications [16]. Polarisation imaging has been used to develop a variety of techniques in computer vision, including surface quality inspection [15],[8],[10], shape recovery [1],[2],[9],[11] and material characterisation [17],[7]. Polarisation can also be used to infer information concerning the reflectance properties of surfaces. For instance, Atkinson and Hancock have shown in [3] how diffuse polarisation can be used to estimate the birectional reflectance function. However, their method is computationally demanding, using simulated annealing to estimate the BRDF. In this paper we take a simpler view of the problem. For fixed light source direction and approximately planar samples, provided that the range of refractive indices for different materials in a scene is limited, the polaristion image allows the angular distribution of reflected or remitted light to be estimated.

Here we exploit this property and parameterise the distribution using spherical harmonics. Vectors of harmonic coefficients are then used to characterise the reflectance distribution on a pixel-by-pixel basis. We can then segment a scene into regions of different reflectance properties using the coefficient vectors. Here we compute the difference in reflectance characteristics using the Mahalanobis distance between coefficient vectors and then use normalised cuts [14] to segment the scene into regions of different material composition. 
The Fresnel theory of light (see [6], [4]) is a quantitative description of reflection and refraction at a smooth boundary between two media. The analysis is relatively straightforward for dielectrics, but the situation is less tractable for metals due to the induction of surface currents by the time varying electromagnetic field of light. In dielectrics, polarisation in remitted light may arise in two different ways. Specular polarisation arises when polarised incident light is reflected from the object surface in the specular direction. In the case of diffuse polarisation, initially unpolarised light is refracted into the surface and the remitted light acquires a spontaneous polarisation due to refraction at the surface.

In both cases the zenith angle of the reflected or remitted light is determined by the degree of polarisation and the azimuth angle determines the polarisation phase angle.

\section{Polarisation Image}

When scattered light is measured through a linear polarising filter, the intensity changes as a sinusoidal function of the polariser angle $\alpha_{p}$ and the transmitted radiance sinusoid (TRS) is given by

$$
I\left(\alpha_{p}\right)=\frac{\left(I_{\max }+I_{\min }\right)}{2}+\frac{\left(I_{\max }-I_{\min }\right)}{2} \cos \left(2 \alpha_{p}-2 \phi\right)
$$

where $I_{\max }$ is the maximum brightness, $I_{\min }$ the minimum brightness and $\phi$ the phase angle. It is more convenient to write the above formula in terms of the mean-intensity

$$
\hat{I}=\frac{1}{2}\left(I_{\max }+I_{\min }\right)
$$

and the degree of polarisation $\rho$, giving

$$
I\left(\alpha_{p}\right)=\hat{I}\left(1+\rho \cos \left(2 \alpha_{p}-2 \phi\right)\right)
$$

Suppose that we take $N$ equally spaced polarisation images, so that the polariser angle index is $p=1,2, \ldots, N$. Let

$$
\begin{gathered}
x_{p}=\left(I\left(\alpha_{p}\right)-\hat{I}\right) / \hat{I}, \\
\hat{x}=\frac{1}{N} \sum_{p=1}^{N} x_{p}
\end{gathered}
$$

and

$$
\sigma^{2}=\frac{1}{N} \sum_{p=1}^{N}\left(x_{p}-\hat{x}\right)^{2}
$$

The moments estimators of the three components of the polarisation image are the mean intensity

$$
\hat{I}=\frac{1}{N} \sum_{p=1}^{N} I\left(\alpha_{p}\right)
$$


the polarisation degree

$$
\rho=\sqrt{2 / \pi} \sigma
$$

and the phase angle

$$
\phi=\frac{1}{2} \cos ^{-1}(\langle\hat{x} \cos (2 \alpha)\rangle / \pi \rho)
$$

We use a moment-based method along with least squares fitting to estimate the degree and phase of polarisation in the scattered light. To improve the robustness of our calculation, observations with large deviations (more than $25 \%$ of the TRS amplitude) are not used in the estimation.

From the Fresnel theory it is straightforward to show that the azimuth angle for reflected polarised light or remitted diffusely polarised light is equal to the phase angle $\phi$ [17]. The zenith angle $\theta$ depends on whether the polarisation is specular or diffuse. For diffuse polarisation, the polarisation degree is given by

$$
\rho_{d}=\frac{(n-1 / n)^{2} \sin ^{2} \theta}{2-2 n^{2}-(n+1 / n)^{2} \sin ^{2} \theta+4 \cos \theta \sqrt{n^{2}-\sin ^{2} \theta}}
$$

while the degree of specular polarisation $\rho_{d}$ is given as:

$$
\rho_{s}=\frac{2 \sin ^{2} \theta \cos \theta \sqrt{n^{2}-\sin ^{2} \theta}}{n^{2}-\sin ^{2} \theta-n^{2} \sin ^{2} \theta+2 \sin ^{4} \theta}
$$

where $n$ is the refractive index.

Here we aim to use (1) through (11) to analyse the distribution of relectance from approximately planar samples of different material. Provided we know whether we are measuring the specular polarisation of reflected polarised light, or the diffuse polarisation of remitted initially unpolarised light, then $\theta$ and $\phi$ are the zenith and azimuth angles of light with respect to the surface normal. To do this we assume the range of refractive index is small, and can be treated as a constant. In our experiments we work with the value $n=1.45$, which is typical of a wide range of dielectrics.

\section{Reflectance Distributions}

The observation underpinning this paper is that under the restrictions of local surface planarity and slowly varying refractive index, the polarisation image allows us to measure the distribution of mean intensity $\hat{I}$ with the zenith and azimuth angle of remitted light, $\theta$ and $\phi$. To provide some illustrative motivation, Fig 1 shows a scatter plot of the intensity versus the degree of polarisation and surface azimuth angle for real and plastic leaves. The leaves are approximately planar, and the angle of incidence is approximately 15 degrees. Here we work with initially unpolarised light and use the formula for diffuse reflectance in (10) to estimate the zenith angle from the measured polarisation. There are a number of features to note from the plot. First, the distributions are quite different for the two materials. We attribute this to the fact that natural leaves have a layered sub-surface structure, which affects distribution of remitted light through subsurface refraction according to Snell's law. Artifical leaves do not exhibit such structure. Second, when the refractive index is changed within the known range for dielectrics, there is a small shift in the plots at all zenith angles. Since the shift is uniform, the effect of approximating refractive index in the feature calculations can be neglected. 

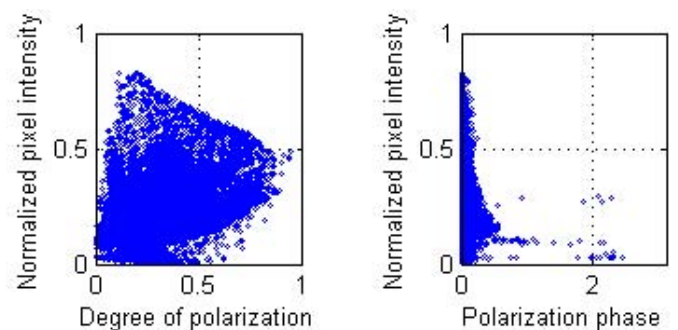

(a) Scatter plots for plastic leaves
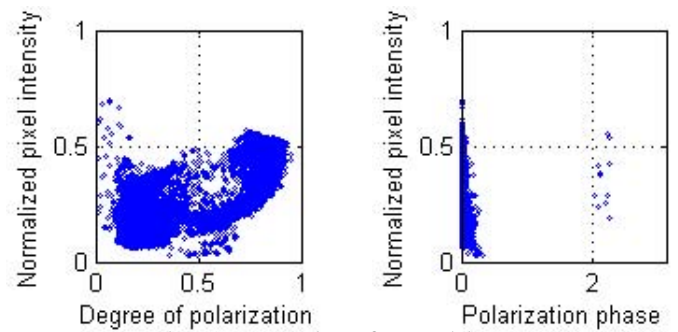

(b) Scatter plots for real leaves

Fig. 1. Scatter plots: The variations in pixel intensity plotted against $\rho$ and $\phi$

\subsection{Calculating Spherical Harmonic Features}

Our idea is to parameterise the distribution of mean intensity as function of the azimuth and zenith angles. The polarisation image consists of a set of triples

$$
P=\left\{\left(\hat{I}_{i}, \rho_{i}, \phi_{i}\right), i=1, \ldots, M\right\}
$$

from which we compute the set

$$
D=\left\{\left(\hat{I}_{i}, \theta_{i}, \phi_{i}\right), i=1, \ldots, M\right\}
$$

using the expression for diffuse polarisation in terms of zenith angle in (10). The distribution of mean image intensity at each pixel is expressed as a function of azimuth and zenith angles. Any such spherically symmetric function $f(\theta, \phi)$ can then be expressed as a weighted sum of the orthonormal basis functions $Y_{l}^{m}$ (called the spherical harmonics of degree $l$ and order $m$ ) as follows:

$$
f(\theta, \phi)=\sum_{l=1}^{\infty} \sum_{m=-l}^{l} a_{l, m} Y_{l}^{m}(\theta, \phi), a \in \mathbb{R}
$$

where $Y_{l}^{m}(\theta, \phi)$ is a function of the associated Legendre polynomials $P_{l}^{m}(z)$ with $z=$ $\cos \theta$, given by

$$
Y_{l}^{m}(\theta, \phi)=\sqrt{\frac{2 l+1}{4 \pi} \frac{(l-m) !}{(l+m) !}} P_{l}^{m}(\cos \theta) e^{i m \phi} .
$$


Using the orthonormality properties of the spherical harmonics, the coefficients are given by

$$
a_{l, m}=\int_{0}^{2 \pi} \int_{0}^{\pi} f(\theta, \phi) Y_{l}^{m}(\theta, \phi) \sin \theta \mathrm{d} \theta \mathrm{d} \phi
$$

From (14), we obtain the following moments estimators of the spherical harmonic coefficients of the mean-intensity distribution.

$$
a_{l, m}=\frac{1}{M} \sum_{i=1}^{M} \hat{I}_{i} Y_{l}^{m}\left(\theta_{i}, \phi_{i}\right)
$$

In practice we estimate the set of coefficients over non-overlapping 10x10 blocks of pixels, and truncate the spherical harmonic expansion at $l=8$ with $m$ varying from $-l$ to $l$. As a result the mean intensity distribution is each pixel block is parameterised by an 81 element vector of spherical harmonic coefficients

$$
A=\left[a_{0,0}, a_{1,-1}, a_{1,0}, a_{1,1}, \ldots, a_{8,8}\right]^{T} .
$$

Estimation of harmonic functions in previous literature includes residual fitting approaches by [13] and [5] and spherical FFT by [12]. We use a MATLAB function to compute the Legendre polynomials and a moments based approach to estimate the coefficients $a_{l, m}$. We divide the image into windows and calculate the average coefficients over each window. The window size is chosen to ensure that the instensity function is a reasonable representation of shape while taking care to not over-smooth the features.

\subsection{Distribution of Information in the Feature Vector}

We aim to use the coefficient vectors for both segmenting and classifying regions in scenes. To this end we commence by computing the variance matrix over blocks of the image. If the image blocks are indexed by $k=1, \ldots, L$ and the $\mathrm{k}$-th block has coefficient $A_{k}$, then the mean coefficient vector is

$$
\hat{A}=\frac{1}{L} \sum_{k=1}^{L} A_{k}
$$

and the covariance matrix is

$$
\Sigma_{A}=\frac{1}{L} \sum_{k=1}^{L}\left(A_{k}-\hat{A}\right)\left(A_{k}-\hat{A}\right)^{T}
$$

The Mahalanobis distance between the coefficient vectors for the blocks indexed $k_{1}$ and $k_{2}$ is

$$
D_{k_{1}, k_{2}}=\left(A_{k_{1}}-A_{k_{2}}\right)^{T} \Sigma_{A}^{-1}\left(A_{k_{1}}-A_{k_{2}}\right)
$$

From the Mahalanobis distance we compute the $L \times L$ block affinity matrix $S$ with elements

$$
S\left(k_{1}, k_{2}\right)=\exp \left[-R D_{k_{1}, k_{2}}\right]
$$

where $R$ is a constant. We segment the polarisation image into regions by recursively applying Shi and Malik's [14] algorithm to the affinity matrix. 


\section{Experiments}

The images are recorded in a darkened room with matte black walls and working surfaces. The studied objects and the camera are positioned on the same axis and a halogen light source (visible spectrum) is positioned at approximately 15 degrees from the viewing axis, to reduce specular reflection. Linear polarising filters are placed in front of the source and the camera. The camera polaroid is rotated through 180 degrees and images are captured with fixed aperture size and exposure time.

Objects studied include fruits, vegetables, natural leaves and plastic leaves. Wolff [16] suggests taking images at polariser orientations 0, 45 and 90, while Atkinson and Hancock [1] use 10 degree intervals of polariser orientations. We choose to record images at 30 degree intervals as a compromise between data collection time and resilience to noise. However with the availability of liquid polarisation cameras data collection time is now a trivial issue.

The polarisation degree captures edges and fine surface texture in unpolarised light and coarse features in polarised light. The polarisation phase captures more surface detail in unpolarised than in polarised light as demonstrated in Fig 2 for a mixed scene of artificial and natural leaves.

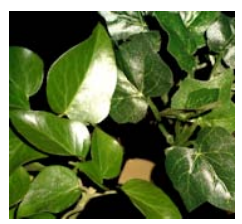

(a) Test scene
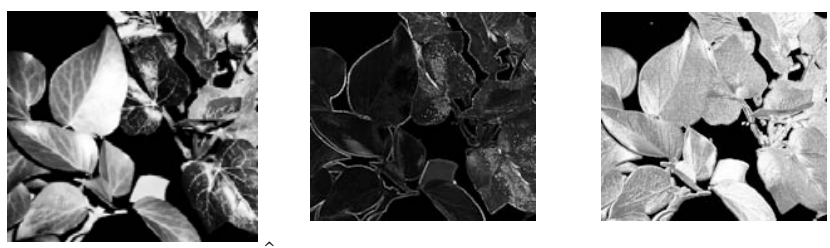

(b) $\hat{I}, \rho$ and $\phi$ in unpolarised light
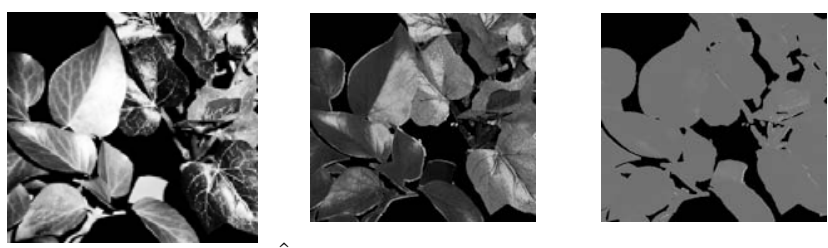

(c) $\hat{I}, \rho$ and $\phi$ in polarised light

Fig. 2. Polarisation image: Grayscale images represent the values of (L-R) mean intensity $\hat{I}$, degree of polarisation $\rho$ and polarisation phase $\phi$ computed for the scene in (a) using images captured in (b) unpolarised incident light and (c) polarised incident light 
We have performed PCA on the coefficient vector covariance matrix $\Sigma_{A}$. The results are shown in Fig 3 which shows the first four principal components which accout for $95 \%$ of the variance. These four components are used to compute a block-by-block feature vector. The features emphasize the vascular structure of real leaves and are weaker in polarised light because the spontaneous polarisation of light on multiple scattering within the real leaf is harder to detect in strongly polarised light.
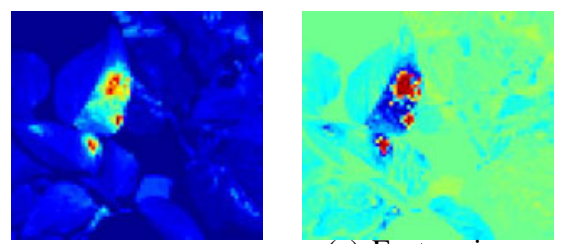

(a) Feature images in unpolarised light
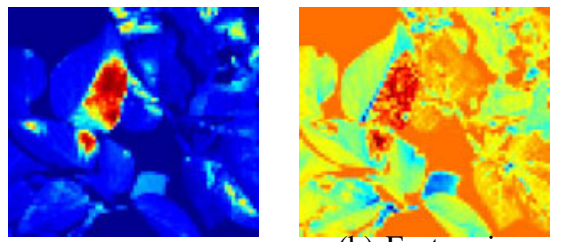

(b) Feature images in polarised light
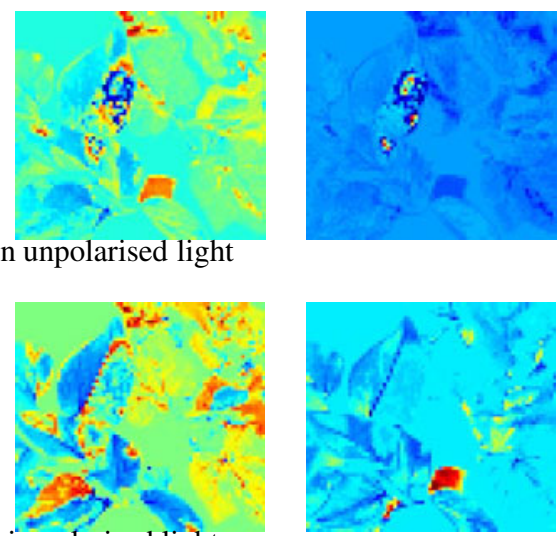

Fig. 3. Feature images (L-R): The first four features for Fig 2(a) calculated from pca-mapped spherical harmonics coefficients

The results of segmenting the scene in Fig 2(a) using normalized graph cuts algorithm from [14] are shown in Fig 4. These results were obtained with 81 features from spherical harmonic coefficients up to degree 8 on a 660x720 image. The affinity matrix was computed using the Mahalanobis distances between the feature vectors in blocks of 10x10 pixels. Specularities cause some difficulty in correct segmentation when using polarised light. Segmentation is better in unpolarised light even in the presence of specularities due to stronger spontaneous polarisation degree and therefore stronger discrimination in coefficient features. The results shown represent a sample of the segmentation results obtained using the proposed method.

The method was also used to successfully segment images taken in natural sunlight. It is well known that light from the sun gets strongly polarised due to scattering by air molecules in the atmosphere. Thus direct sunlight is polarised light. However reflection from object surfaces tends to spontaneously depolarise light. Thus in outdoor settings, images taken in direct and diffuse light can be considered to be under polarised and unpolarised states of incident light. Figure 5(a) contains a section of plastic leaves imaged among the branches of shrub in diffuse sunlight and 5(c) captures camouflage in the midst of a hedge in direct sunlight.

The outdoor scene in Figure $5(\mathrm{a})$ consists of an image $336 \times 384$ pixels in size and is divided into blocks of size $24 \times 24$ for spherical harmonic coefficient calculation. The harmonic expansion is truncated at order 30. Out of 112 blocks, 66 are classified correctly and 16 blocks contain both materials, giving a segmentation accuracy of $71 \%$. 

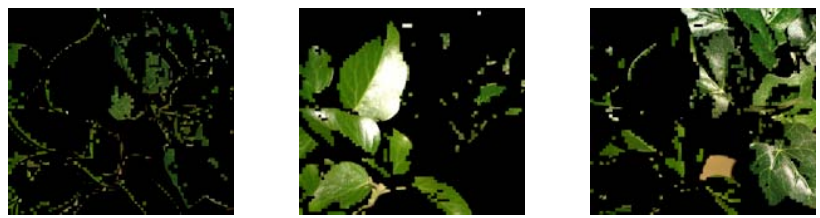

(a) Segmentation in unpolarised light
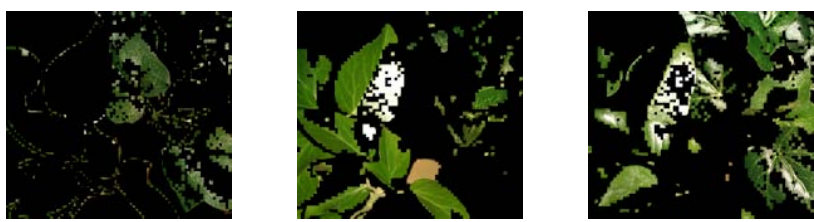

(b) Segmentation in polarised light

Fig. 4. Segmentation: The image in Fig 2(a) is segmented using normalized cuts into (L-R) background, natural leaves and plastic leaves

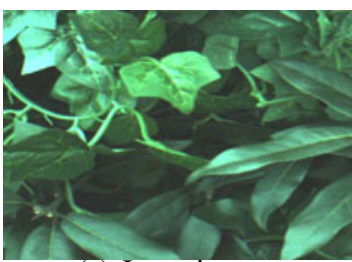

(a) Input image

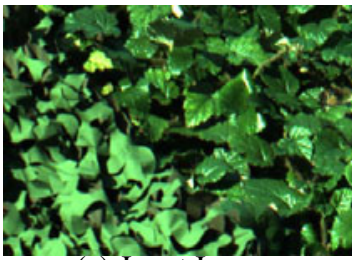

(c) Input lmage
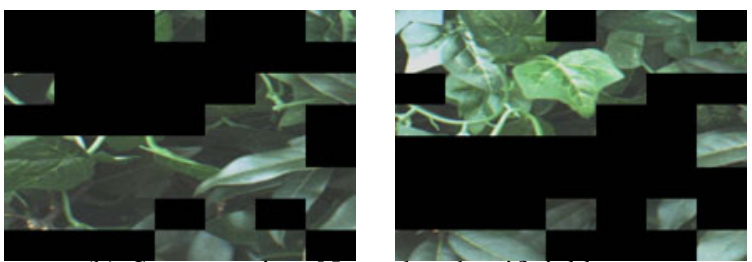

(b) Segmentation: Natural and artificial leaves

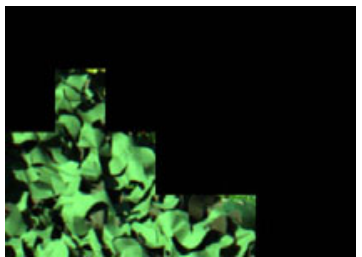

(d) Segmentation: Camouflage and natural leaves

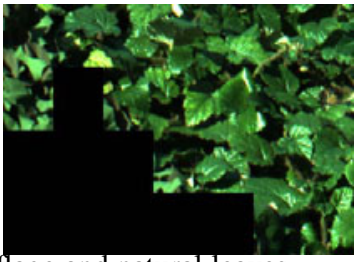

Fig. 5. Outdoor scenes : Imaged scenes include (a) real and plastic leaves in diffused sunlight (c) natural leaves and camouflage in direct sunlight. (b) and (d) show the segmentation results using the proposed method.

For the $370 \times 420$ image in Figure $5(\mathrm{c})$, a block size of $66 \times 66$ for feature generation produced the best segmentation performance. Out of 28 blocks, 2 blocks are classified incorrectly, and 3 blocks contain sections of both materials. Assuming that all blocks containing more than one material are incorrectly classified gives an accuracy of $82 \%$ while a more optimistic estimation gives an accuracy of $93 \%$.

Segmentation results were tested for different values of window size and orders of expansion. The window size that produces optimal segmentation is found to vary from scene to scene. It is noted that for the window size that produces optimal segmentation, 


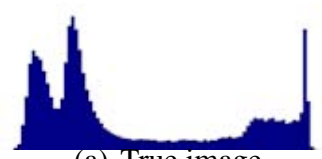

(a) True image

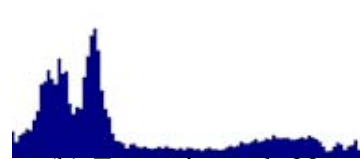

(b) Expansion at $1=20$

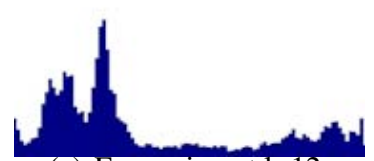

(c) Expansion at $\mathrm{l}=12$

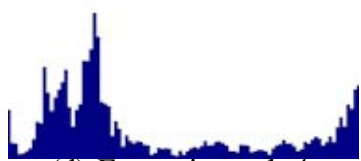

(d) Expansion at $1=4$

Fig. 6. Histograms: (a) shows an image histogram and (b) to (d) show histograms of reconstructed pixel intensities with exapnsions upto $l=20,12$ and 4

spherical harmonic expansion truncated at order 12 gives the desired segments and the results are stable over multiple runs. Adding higher order terms to the feature vector does not improve performance noticeably. This implies that most of the key feature information is contained in low order harmonics, up to order 12. For other window sizes however the normalized cuts seem to produce unpredictable results. Further investigation to analyze the factors that affect the accuracy of segmentation presents a topic for further research in this method.

The accuracy of our intensity function can be checked by reconstructing the intensity at each pixel as a spherical harmonic expansion using (12). The histograms in Fig.fig:histo compare the original histogram (a) with reconstructions (b) - (d) using coefficients of degree 20, 12 and 4, for a patch of the scene in Fig 2(a). The reconstruction error shows a drop with increase in degree of expansion, as expected. The resolution of reconstruction however is limited by the choice of window size in coefficient calcuation. The reconstructed images show a smoothing effect attributed to the truncation of spherical harmonic expansion and the windowing in calculation of coefficients.

\section{References}

1. Atkinson, G., Hancock, E.R.: Recovery of surface orientation from diffuse polarization. IEEE Transactions on image Processing 15(6), 1653-1664 (2006)

2. Atkinson, G., Hancock, E.R.: Polarization-based surface reconstruction via patch matching. Computer Analysis of Images and Patterns, 466-473 (2007)

3. Atkinson, G., Hancock, E.R.: Two-dimensional brdf estimation from polarisation. Computer Vision and Image Understanding 111(2), 126-141 (2008)

4. Born, M., Wolf, E.: Principles of Optics, 7th (expanded) edn. Cambridge University Press, Cambridge (1999)

5. Chung, M.K., Dalton, K.M., Davidson, R.J.: Tensor-based cortical surface morphometry via weighted spherical harmonic representation. IEEE Transactions on Medical Imaging 27(8), 1143-1151 (2008)

6. Hecht, E.: Optics, 4th edn. Addison-Wesley, Reading (2002)

7. Jones, B.F., Fairney, P.T.: Recognition of shiny dielectric objects by analyzing the polarization of reflected light. Image and Vision Computing Journal 7 (1989) 
8. Meriaudeau, F., Ferraton, M., Stolz, C., Morel, O., Bigué, L.: Polarization imaging for industrial inspection, vol. 6813 (2008)

9. Miyazaki, D., Kagesawa, M., Ikeuchi, K.: Transparent surface modeling from a pair of polarization images. IEEE Transactions on Pattern Analysis and Machine Intelligence 26(1), 73-82 (2004)

10. Morel, O., Stolz, C., Meriaudeau, F., Gorria, P.: Active lighting applied to three-dimensional reconstruction of specular metallic surfaces by polarization imaging. Applied Optics 45(17), 4062-4068 (2006)

11. Rahmann, S., Canterakis, N.: Reconstruction of specular surfaces using polarization imaging. In: Proc. IEEE Conf. Computer Vision and Pattern Recognition, vol. 1, pp. 149-155 (2001)

12. Saupe, D., Vranić, D.V.: 3-d model retrieval with spherical harmonics and moments. In: Proceedings of the 23rd DAGM-Symposium on Pattern Recognition, pp. 392-397. Springer, Heidelberg (2001)

13. Shen, L., Ford, J., Makedon, F., Saykin, A.: A surface-based approach for classification of 3d neuroanatomic structures. Intelligent Data Analysis 8(6), 519-545 (2004)

14. Shi, J., Malik, J.: Normalized cuts and image segmentation. IEEE Transactions on Pattern Analysis and Machine Intelligence 22(8), 888-905 (2000)

15. Sun, G., Onoichenco, E., Fu, Y., Liu, Y., Amell, R., McCandless, C., Reddy, R., Kumar, G., Guest, M.: High-throughput polarization imaging for defocus and dose inspection for production wafers, vol. 6518 (2007)

16. Wolff, L.B.: Polarization vision: a new sensory approach to image understanding. Image and Vision Computing 15, 81-93 (1997)

17. Wolff, L.B., Boult, T.E.: Constraining object features using a polarisation reflectance model. IEEE Transactions on Pattern Analysis and Machine Intelligence 13(7), 635-657 (1991) 